ISSN 2447-9071

doi https://doi.org/10.36414/rbmc.v6i16.70

Contato para correspondência: Roberpaulo Anacleto Neves

E-mail:

hroberpaulo.neves@faculdadepm.edu.br

Conflito de interesse: Não

Financiamento: Recursos próprios

Recebido: $19 / 11 / 2020$

Aprovado: 26/11/2020

\section{Análise espacial e epidemiológica dos casos de Síndrome Respiratória Aguda Grave por influenza A/H1N1 no estado de Goiás no período de 2016 a 2018}

\section{Spatial and epidemiological analysis of cases of Severe Acute Respiratory Syndrome caused by influenza $\mathrm{A} / \mathrm{H} 1 \mathrm{~N} 1$ in the state of Goiás from 2016 to 2018}

Luís Gustavo Silva Ribeiro', Jhefferson Einstein Rodrigues Rezende' ${ }^{1}$ Roberpaulo Anacleto Neves ${ }^{1,2}$

${ }^{1}$ Pontifícia Universidade Católica de Goiás - PUC Goiás

${ }^{2}$ Faculdade da Polícia Militar - FPM

\section{Resumo}

Este estudo teve por objetivo realizar uma análise espacial e epidemiológica das notificações de casos de Síndrome Respiratória Aguda Grave (SRAG) por influenza A/H1N1 no estado de Goiás no período de 2016 a 2018. Trata-se de umestudo transversal analítico de prevalência com dados obtidos através do Instituto Brasileiro de Geografia e Estatística (IBGE) e EXTRANET e os limites espaciais através dos sites do Laboratório de Processamento de Imagens e Geoprocessamento (LAPIG) e Pastagem. Dados foram tabulados no Microsoft Office Excel 2010. Análise estatística feita com o teste Qui-quadrado, adotando um nível de significância de $95 \%$. Gráficos e tabelas confeccionados utilizando-se o programa estatístico Bioestat 5.3 e GraphPad Prism 7.0 e mapas confeccionados com o software ArcGis. Em 2016, entre os 246 municípios do estado de Goiás, 73 foram positivos para casos de SRAG por H1N1. Das cinco variáveis socioeconômicas analisadas (PIB per capita, IDH, IDEB, salário médio mensal e $N^{\circ}$ de unidades de saúde), foram encontradas quatro com significância estatística: PIB per capita, IDH, salário médio mensal e $N^{\circ}$ de unidades de saúde. Em 2017, apenas 2 municípios apresentaram casos e a única variável com significância estatística foi o $N^{\circ}$ de unidades de saúde. Em 2018, foram 83 municípios positivos para a presença de casos e quatro variáveis com significância estatística:IDH, salário médio mensal e $N^{\circ}$ de unidades de saúde. Concluiu-se que houve relação entre o surgimento de casos de SRAG por H1N1 nos municípios de Goiás no período de 2016 a 2018 com o PIB per capita, IDH, salário médio mensal e $N^{\circ}$ de unidades de saúde, o que não foi observado como IDEB, já que essa variável nãoapresentou significânciaestatística. Observou-se também que houve umapredominância dos casos na região central do estado de Goiás.

Palavras-Chave: H1N1, Epidemiologia de SRAG, Goiás.. 
(GDP per capita, $H D I$ I IDEB, average monthly salary and $N^{\circ}$ of health units), four with statistical significance were found: GDP per capita, HDI, average monthly wage and $N^{\circ}$ of health units. In 2017, only 2 municipalities had cases and the only variable with statistical significance was the number of health units. In 2018, 83 municipalities were positive for the presence of cases and four variables with statistical significance: $\mathrm{HDl}$, average monthly salary and $N^{\circ}$ of health units. It was concluded that there was a relationship between the appearance of cases of SARS by H1N1 in the municipalities of Goiás in the period from 2016 to 2018 with GDP per capita, HDI, average monthly salary and $N^{\circ}$ of health units, which was not observed with IDEB, since this variable did not present statistical significance. It was also observed that there was a predominance of cases in the central region of the state of Goiás.

Keywords: H1N1, Epidemiology of SARS, Goiás.

\section{Introdução}

A Síndrome Respiratória Aguda Grave (SRAG) se configura como um distúrbio respiratório infeccioso que evolui rapidamente para uma insuficiência respiratória e ocasionou a primeira pandemia do século XXI'. A princípio foi identificada na província de Guangdong na China em 2002 e em 2003, onde foi descoberto o vírus que a desencadeou, chamado de Coronavírus (SARS-CoV). Mais de 8.000 casos foram confirmados em $2003^{2}$.

O quadro clínico se assemelha a uma síndrome gripal (SG) caracterizado por febre, mal-estar, mialgia e diarreia, além de tosse seca não produtiva e dispneia. Por esse motivo não pode ser identificada clinicamente nos primeiros dias de infecção. Suas características clínicas estão associadas a pelo menos um dos seguintes sinais e sintomas: dispneia, saturação de $\mathrm{SO} 2<95 \%$ em ar ambiente, desconforto respiratório ou aumento da frequência respiratória, piora nas condições clínicas das doenças de base e hipotensão. Em crianças, além do quadro clínico acima descrito podem ser observados batimentos deasa de nariz, cianose, tiragem intercostal, desidratação e inapetência. Para considerar um paciente portador deSRAG, de acordo com o protocolo de vigilância de influenza, esse deve apresentar-se com dispneia, tosse e febre $>38^{\circ} \mathrm{C}$ podendo estar acompanhados ou não de manifestações gastrointestinais ou de outros sinais de gravidade ${ }^{1,3,4}$.

A saber disso, em 2009 uma nova pandemia de SRAG surgiu na Califórnia (EUA) e no México, se alastrando para todo o planeta. Um novo vírus influenza A subtipo H1N1 foi considerado o responsável pelo ocorrido e a doença ficou popularmente conhecida como "gripe suína". O primeiro caso de H1N1 no Brasil foi confirmado em maio de 20095. O Ministério da Saúde (MS), logo após a pandemia ocorrida em 2009, preconizou a notificação compulsória exclusivamente dos casos considerados graves de influenza. Isso logo mudou quando em 2012 a ficha de notificação, antes utilizada apenas para casos de influenza, foi alterada e seu monitoramento foi redirecionado, pela vigilância epidemiológica, para a Síndrome Respiratória Aguda Grave (SRAG) ${ }^{3}$.
O vírus da influenza $A$ pertence à família Ortomixovírus e é mais prevalente e responsável por grande número de mortes em humanos dos subtipos da influenza. Segundo o Ministério da Saúde (MS), essa doença resulta em 3 a 5 milhões de casos graves e 250.000 a 500.000 mortes, anualmente ${ }^{6,7}$. Os grupos de risco de desenvolvimento agudo da doença incluem idosos, gestantes, pacientes imunocomprometidos, pessoas com doenças crônicas, crianças menores de 5 anos de idade e profissionais da saúde. A transmissão é, majoritariamente, por gotículas, mãos contaminadas e inoculação direta do vírus e espaços fechados e aglomerados de pessoas auxiliam na disseminação dessa enfermidade ${ }^{2,6}$.

O MS implantou a Vigilância da Influenza no Brasil que tem como componente a Vigilância Universal de SRAG. Esta tem como objetivo rastrear o comportamento da influenza no país e dessa forma orientar o Ministério da Saúde e Secretarias de Saúde Estaduais e Municipais na tomada de decisões importantes referentes a essa questão de saúde. Esse rastreio é feito por meio do monitoramento dos casos hospitalizados de SRAG e óbitos ${ }^{8}$.

Os dados epidemiológicos brasileiros de Síndrome Respiratória Aguda Grave (SRAG) por influenza A/H1N1 foram coletados por um monitoramento de 52 semanas epidemiológicas do ano de 2017. Esse rastreio foi realizado pela Secretaria de Vigilância em Saúde - Ministério da Saúde por meio de formulários padronizados e inseridos nos sistemas de informação online: SIVEP-Gripe e SINAN Influenza Web?

Nesse ano foram notificados 22.499 casos de SRAG em todo o Brasil, sendo que 18.036 (80,2\%) foram com amostra processada. Destas amostras, apenas 14,9\% (2.691/18.036) foram classificadas como SRAG por influenza e dentre estes, um total de 48 (1,8\%) eram influenza $A(H 1 N 1)$. Em relação aos óbitos nas mesmas 52 semanas epidemiológicas de 2017 foram notificados 3.277 casos de óbitos por SRAG, correspondendo a $14,6 \%$ (3.277/22.499) do total de casos. Dentre o total de óbitos notificados, $498(15,2 \%)$ confirmaram-se para vírus influenza, 
sendo $12(2,4 \%)$ casos decorrentes de influenza $A(\mathrm{H} 1 \mathrm{~N} 1)^{9}$.

No estado de Goiás no ano de 2018 foram notificados 1561 casos de SRAG nas 41 semanas epidemiológicas analisadas, o que representa um crescimento de $134.74 \%$ em relação ao ano anterior. Destes casos, 465 (29.81\%) foram provocados por influenza e um total de 396 (25.38\%) tiveram como causa específica a influenza $A / H 1 N 1$. A respeito dos casos de óbitos nesse mesmo ano de 2018 nas 41 semanas analisadas foram notificados 234 óbitos, o que representa um crescimento de $100 \%$ em relação ao ano anterior. Destes casos, 84 (35.9\%) foram provocados por SRAG por influenza e um total de 75 (32.05\%) tiveram como causa específica a SRAG por influenza $\mathrm{A} / \mathrm{H} 1 \mathrm{~N} 1^{3}$.

Em relação às Regionais de Saúde, a que apresentou maior número de casos de SRAG por influenza A/H1N1 foi a Central com um total de 172 casos, tendo Goiânia como o município com maior incidência (131 casos). O número de óbitos segue a mesma linha, tendo a Regional Central com 34 óbitos e o município de Goiânia com a maior incidência (21 óbitos) 3 .

A justificativa se baseia na preocupação com o difícil controle de casos de Síndrome Respiratória Aguda Grave (SRAG) causada por influenza A/H1N1. Além disso, uma análise espacial e epidemiológica dos casos de SRAG no estado de Goiás por meio das notificações de casos no período de 2016 a 2018, pode auxiliar, além do esclarecimento da relação dessa doença com as epidemias de $\mathrm{H} 1 \mathrm{~N} 1$ nesse período, também na compreensão de suas causas e até mesmo na implementação de estratégias de detecção e prevenção.

A disseminação dessas informações de saúde pode permitir a tomada de decisões baseadas em evidências e a programação das ações de saúde, como campanhas de vacinação. Assim, o estudo torna-se relevante devido à escassez de pesquisas deste caráter sobre SRAG por influenza A/H1N1 e devido ao aumento da incidência dos casos de tal doença no estado de Goiás neste período. São os dados de mais fácil acesso e disponíveis para análise situacional dos casos de SRAG notificados sendo, também, as informações mais recentes e atualizadas que constam na base de dados informatizada da Secretaria de Estado da Saúde de Goiás (EXTRANET). O objetivo deste estudo foi realizar uma análise espacial e epidemiológica das notificações de casos de Síndrome Respiratória Aguda Grave (SRAG) por influenza A/ H1N1 no estado de Goiás no período de 2016 a 2018.

\section{Métodos}

Trata-se de um estudo transversal analítico de prevalência, em que houve análise espacial e epidemiológica dos casos de Síndrome Respiratória Aguda Grave (SRAG) por influenza A/ H1N1 no estado de Goiás no período de 2016 a 2018 através de algumas variáveis socioeconômicas, como: Produto Interno
Bruto (PIB) per capita, Índice de Desenvolvimento Humano (IDH), Índice de Desenvolvimento da Educação Básica (IDEB), Salário Médio Mensal dos Trabalhadores (SMM) e Número de Unidades de Saúde (NUS). A coleta de dados foi feita a partir do Instituto Brasileiro de Geografia e Estatística (IBGE) e do EXTRANET e os limites espaciais foram obtidos através dos sites do Laboratório de Processamento de Imagens e Geoprocessamento (LAPIG) e Pastagem e os mapas confeccionados com auxílio do software ArcGis.

Os pacientes foram avaliados de acordo com os critérios de inclusão do estudo, que são: pacientes que possuíam Síndrome Respiratória Aguda Grave (SRAG) causada por influenza A/H1N1. Os critérios de exclusão foram pacientes que não possuíam Síndrome Respiratória Aguda Grave ou que possuíam tal patologia sem ter como causa a influenza A/H1N1.

Os dados e limites espaciais foram tabulados e processados pelo programa Microsoft Office Excel 2010 e, a seguir, foram gerados mapas no formato Shapefiles (SHP) em conjunto com o software ArcGis. A análise estatística foi realizada comparando as variáveis categóricas por meio do teste Qui-quadrado. Por meio de regressão linear foi analisado se houve tendência de crescimento da doença no período estudado. O nível de significância adotado foi de $95 \%$. Foi utilizado o programa estatístico Bioestat 5.3 e GraphPad Prism 7.0 para confecção dos gráficos e tabelas.

Como o estudo foi realizado a partir de dados de domínio público informatizados, não foi necessária a apreciação do Comitê de Ética em Pesquisa (CEP), segundo a resolução CNS 466/2012.

\section{Resultados}

Em 2016, dos 246 municípios do estado de Goiás, 73 foram positivos para casos de SRAG por H1N1. Das cinco variáveis socioeconômicas analisadas (PIB per capita, IDH, IDEB, SMM e número de unidades de saúde) foram encontradas quatro com significância estatística para a ocorrência dos casos, são elas: PIB per capita $(p=0,0022), \operatorname{IDH}(p=0,0002), \operatorname{SMM}(p=0,0001)$ e NUS ( $p=0,0001)$. Em 2017 apenas 2 municípios apresentaram casos e a única variável com significância estatística foi o número de unidades de saúde ( $p=0,0216)$. Em 2018 foram 83 municípios positivos para a presença de casos e quatro variáveis com significância estatística, são elas: IDH ( $p=0,0003)$, salário médio mensal $(p=0,0019)$ e número de unidades de saúde ( $p$ $=0,0001$ ) (Tabela 1). 
Tabela 1. Relação entre a ocorrência de casos de SRAG por H1N1 nos municípios de Goiás e as variáveis socioeconômicas, utilizando o teste Qui-quadrado, entre 2016 e 2018.

\begin{tabular}{|c|c|c|c|c|c|c|}
\hline \multirow{2}{*}{ PIB per capita } & \multicolumn{2}{|c|}{ No casos 2016} & \multicolumn{2}{|c|}{ No casos 2017} & \multicolumn{2}{|c|}{ № casos 2018} \\
\hline & Positivos & Negativos & Positivos & Negativos & Positivos & Negativos \\
\hline$<22.683,52$ & 38 & 125 & 1 & 162 & 53 & 110 \\
\hline$\geq 22.683,52$ & 35 & 48 & 1 & 82 & 30 & 53 \\
\hline$p$ & & 0,0022 & & 0,6253 & & 0,5692 \\
\hline IDH & Positivos & Negativos & Positivos & Negativos & Positivos & Negativos \\
\hline$<0,7$ & 26 & 106 & 0 & 132 & 31 & 101 \\
\hline$\geq 0,7$ & 47 & 67 & 2 & 112 & 52 & 62 \\
\hline$p$ & & 0,0002 & & 0,1265 & & 0,0003 \\
\hline
\end{tabular}

$\begin{array}{lcccccc}\text { IDEB } & \text { Positivos } & \text { Negativos } & \text { Positivos } & \text { Negativos } & \text { Positivos } & \text { Negativos } \\ <4,7 & 28 & 61 & 0 & 89 & 29 & 60 \\ \geq 4,7 & 45 & 112 & 2 & 155 & 54 & 103 \\ p & & 0,6443 & & 0,285 & & 0,7729 \\ & & & & & & \\ \text { SMM } & \text { Positivos } & \text { Negativos } & \text { Positivos } & \text { Negativos } & \text { Positivos } & \text { Negativos } \\ <2 & 17 & 109 & 0 & 126 & 31 & 95 \\ \geq 2 & 56 & 64 & 2 & 118 & 52 & 68 \\ p & & <0,0001 & & 0,1457 & & 0,0019\end{array}$

\begin{tabular}{lcccccc} 
NoUS & Positivos & Negativos & Positivos & Negativos & Positivos & Negativos \\
$<8$ & 31 & 147 & 0 & 178 & 35 & 143 \\
$\geq 8$ & 42 & 26 & 2 & 66 & 48 & 20 \\
$p$ & & $<0,0001$ & & 0,0216 & & $<0,0001$ \\
\hline
\end{tabular}

Legenda: PIB: Produto Interno Bruto; IDH: Índice de Desenvolvimento Humano; IDEB: Índice de Desenvolvimento da Educação Básica; SMM: Salário Médio Mensal (salário mínimo) NUS: Número de unidades de saúde; p: valor de $p$.

Foram identificados os dez municípios do estado de Goiás com maior número de casos por 100 mil habitantes, nos anos de 2016, 2017 e 2018. Em 2016, destacaram-se: São Patrício (96,61/100.000), Goianira (65,58/100.000), Jandaia (63,95/100.000), Caldazinha (54,58/100.000), Abadia de Goiás (49,67/100.000), Ouvidor (47,31/100.000), Aparecida do Rio
Doce (39,77/100.000), Pilar de Goiás (39,54/100.000), Panamá $(36,87 / 100.000)$ e Iporá $(31,03 / 100.000)$. Destes, seis situam-se na região central: São Patrício, Goianira, Caldazinha, Abadia de Goiás, Pilar de Goiás e Iporá. Em 2017 apenas os municípios de Goianésia $(1,48 / 100.000)$ e Goiânia $(0,2 / 100.000)$ apresentaram casos, estando os dois situados na região central do estado. Em 2018, foram: Ivolândia (232,82/100.000), Novo Brasil (92,53/100.000), Ceres (68,07/100.000), Jaupaci (66,57/100.000), Buriti Alegre (42,1/100.000), Carmo do Rio Verde (40,95/100.000), Diorama (39,27/100.000), Damolândia (34,25/100.000), Arenópolis $(33,21 / 100.000)$ e Iporá $(31,03 / 100.000)$. Destes, sete situam-se na região central: Ivolândia, Novo Brasil, Ceres, Jaupaci, Carmo do Rio Verde, Damolândia e Iporá (Figura 1).

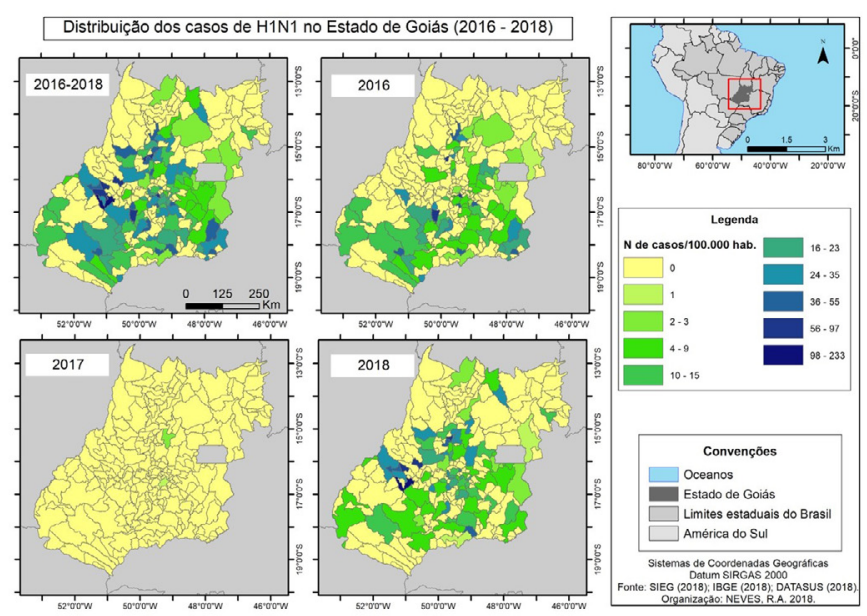

Figura 1. Distribuição do número de casos de SRAG por H1N1 por 100.000 habitantes no estado de Goiás, no período de 2016 a 2018.

Este trabalho também se propôs a relacionar a distribuição de casos de SRAG por H1N1 em Goiás com as variáveis socioeconômicas. Assim, foi relacionado o número de casos por 100.000 habitantes com as variáveis: IDH, IDEB, № US e PIB per capita. Foi possível avaliar uma maior ocorrência de casos nos municípios que apresentaram um IDH elevado $(\geq 0,7)$. A Figura 2 ilustra a distribuição de casos de SRAG por H1N1 nos municípios de Goiás referente ao IDH nos anos de 2016, 2017 e 2018. 


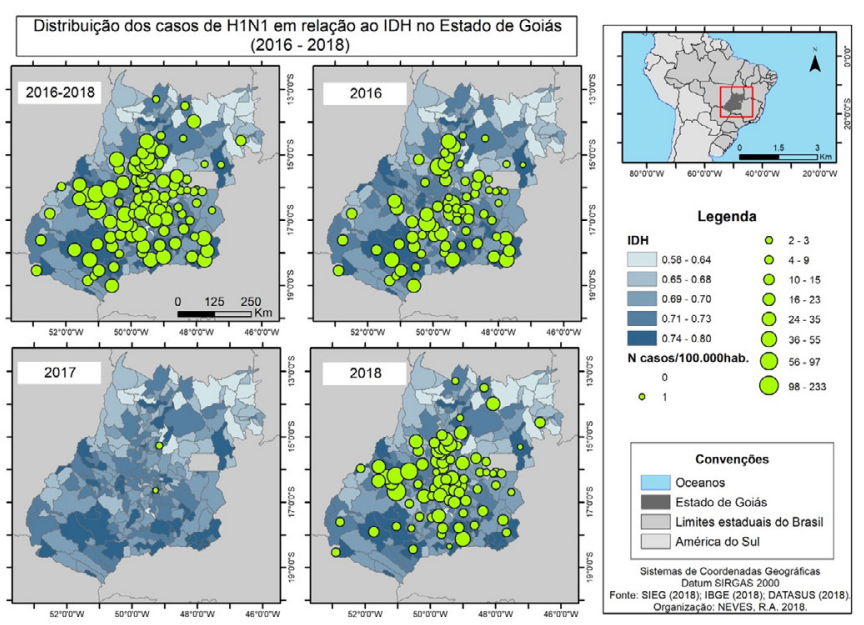

Figura 2. Distribuição do número de casos de SRAG por H1N1 por 100.000 habitantes em relação ao IDH de cada município no estado de Goiás, no período de 2016 a 2018.

Em relação ao IDEB, foi possível avaliar uma maior ocorrência de casos nos municípios que apresentaram um valor $\geq 4,7$. A Figura 3 ilustra a distribuição de casos de SRAG por H1N1 nos municípios de Goiás referente ao IDEB nos anos de 2016, 2017 e 2018.

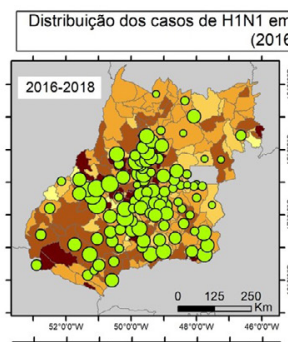
(2016 - 2018)
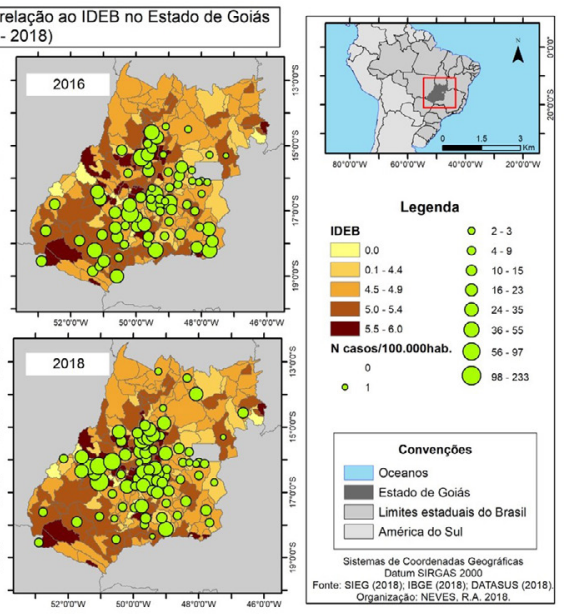

Figura 3. Distribuição do número de casos de SRAG por H1N1 por 100.000 habitantes em relação ao IDEB de cada município no estado de Goiás, no período de 2016 a 2018.

Em relação ao PIB per capita e ao número de unidades de saúde foi possível observar uma menor ocorrência de casos nos municípios que obtiveram um valor de PIB per capita $\geq$ 22.683,52 e uma maior ocorrência de casos nos municípios com um número de unidades de saúde $\geq 8$. A Figura 4 ilustra a distribuição de casos de SRAG por H1N1 nos municípios de Goiás referente ao PIB per capita e o número de unidades de saúde nos anos de 2016, 2017 e 2018.
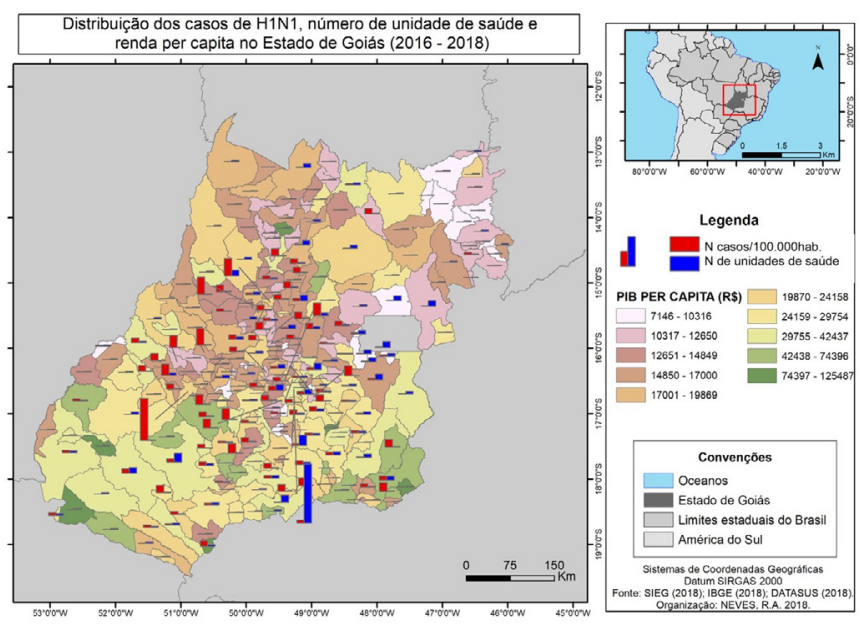

Figura 4. Distribuição do número de casos de SRAG por H1N1 por 100.000 habitantes em relação ao número de unidades de saúde e o PIB per capita de cada município no estado de Goiás, no período de 2016 a 2018.

\section{Discussão}

A partir de uma análise espacial e epidemiológica da incidência da SRAG por H1N1 nos municípios de Goiás entre os anos de 2016 a 2018, verificou-se que houve um predomínio da região central do estado entre os dez municípios com maior número de casos por 100 mil habitantes. Esse predomínio intensificou-se com o aumento do número de casos visto em 2018 em relação ao ano de 2016, no entanto, em 2018, apenas o município de Iporá continuou entre os dez. Um estudo publicado por Guedes em 201110, realizou uma análise epidemiológica da situação da influenza A/H1N1 nos anos de 2009 e 2010 no estado de Goiás e foi observado que a maior incidência do surto do vírus H1N1 foi na região Central. Tal fato foi explicado pela facilidade de acesso a essa região o que permite um fluxo circulante de pessoas muito grande, favorecendo assim a disseminação do vírus.

Ao analisar o IDH foi possível concluir que houve uma relação diretamente proporcional dessa variável com a incidência de SRAG porH1N1 nos municípios do estado de Goiás. Observou-se uma incidência maior de casos nos municípios que apresentaram um IDH alto $(\geq 0,7) 11$. Uma análise semelhante foi realizada em um estudo publicado por Rodriguez em 201012, em que foi avaliada a associação do IDH com a epidemiologia da de H1N1 nos países da América Latina. Foi observado nesse estudo que os países com melhores condições de vida tinham uma morbidade consideravelmente maior devido à influenza $\mathrm{A} / \mathrm{H} 1 \mathrm{~N} 1$, o que foi 
explicado pelo maior fluxo de pacientes e maior acesso à saúde da população afetada.

A analise do número de unidades de saúde por município demonstrou importante relação entre a quantidade de unidades existentes e a notificação de casos. Observou-se que municípios com uma maior concentração de unidades de saúde por habitantes apresentaram também uma maior incidência de casos entre os três anos estudados. Segundo um estudo publicado por Montalvão em 201713, vem sendo implementado no Brasil desde o ano 2000 o Sistema Nacional de Vigilância da Influenza. Esse sistema tem como objetivo realizar o monitoramento dos casos de síndrome gripal e da circulação dos principais vírus responsáveis por SRAG na comunidade. A partir de 2012 houve a inclusão de todas as Unidades Federativas brasileiras como participantes da rede sentinela para Influenza, que é composta por unidades de saúde definidas pelos gestores e técnicos dos municípios, estados e Distrito Federal. Dessa maneira entendese que as unidades de saúde são responsáveis pelo rastreio e notificação dos casos de SRAG, o que deixa claro a relação diretamente proporcional entre o número de unidades por município e a quantidade de casos notificados.

Em relação ao salário médio mensal dos trabalhadores de cada município pesquisado, foi possível observar que também houve uma maior incidência de casos naqueles municípios que obtiveram maior média de salário mensal ( $\geq 2$ salários mínimos) nos três anos. Um estudo publicado por Victor em 201414, ao analisar os fatores associados à vacinação contra Influenza A/ H1N1 em idosos, concluiu que entre aqueles que haviam sido vacinados, houve uma predominância da população que tinha renda mensal entre um e dois salários mínimos. Tal evidência justifica a maior incidência de SRAG por $\mathrm{H} 1 \mathrm{H} 1$ em indivíduos que estão fora dessa faixa salarial, pois entende-se que estes estão mais sujeitos à doença por apresentarem uma menor adesão à vacinação contra Influenza $\mathrm{A} / \mathrm{H} 1 \mathrm{~N} 1$.

Quanto ao PIB per capita, mesmo que tenha apresentado uma significância estatística apenas no ano de 2016, foi possível observar uma relação inversamente proporcional com a incidência de casos, diferentemente do que foi encontrado para as outras variáveis analisadas. Houve maior incidência dos casos nos municípios que apresentaram um PIB per capita abaixo da média encontrada referente a todos os municípios de Goiás. Segundo uma análise, publicada por Oliveira em 201415, que analisou a cobertura da vacina anti-influenza em idosos de municípios do estado do Rio Grande do Sul, observou-se que houve uma relação diretamente proporcional entre o do PIB dos municípios do Rio Grande do Sul e a cobertura vacinal. Entre os anos de 2006 e 2010 pode-se observar um crescimento conjunto de ambos, o que pode ser explicado pelo fato de maio- res finanças tenderem a ter uma população melhor instruída e estarem associadas a um investimento maior no setor primário de sistema de saúde.

O IDEB foi a única variável que não apresentou significância estatística em nenhum dos três anos estudados, o que leva a concluir que esse índice não apresentou relação com a incidência de casos de SRAG por H1N1 nos municípios de Goiás. No entanto, uma pesquisa, publicada por Pereira em 201316, realizou uma análise dos motivos que levaram gestantes a não se vacinarem contra $\mathrm{H} 1 \mathrm{~N} 1$ e concluiu-se que a falta de informação foi uma das principais razões que levaram a tal recusa. Baseando-se nisso, pode-se entender que que um baixo índice de desenvolvimento educacional pode apresentar influência na epidemiologia de SRAG por H1N1 devido a uma deficiência na cobertura vacinal em populações carentes de informação.

\section{Conclusão}

Foi possível concluir, a partir da análise estatística dos dados coletados, que houve uma relação diretamente proporcional entre o surgimento de casos de SRAG por H1N1 nos municípios de Goiás nos anos de 2016, 2017 e 2018 com o PIB, IDH, salário médio mensal e número de unidades de saúde. No entanto, não foi observada relação do IDEB com o surgimento de casos nos municípios de Goiás, já que essa variável não apresentou significância estatística em nenhum dos três anos analisados. Pode-se concluir também, a partir de uma análise espacial e epidemiológica, que houve uma significativa predominância proporcional de casos de SRAG por H1N1 na região central do estado de Goiás. Tal fato se baseia na facilidade de acesso e grande fluxo de pessoas nessa região, o que contribuiu para um incremento nas notificações dessa localização.

\section{Referências}

1. Treanor JJ. Influenza Viruses, Including Avian Influenza and Swine Influenza. In: Mandell GL, Bennett JE, Dolin R. Principles and practice of infectious diseases. 7. ed. Philadelphia: Churchill Livingstone - Elsevier; 2010.

2. World Health Organization. Summary of probable SARS cases with onset of illness from 1 November 2002 to 31 July 2003.23 September 2003. Available at: http://www. who.int/csr/sars/country/2019_06_10/en (accessed 06 October 2019).

3. Goiás. Boletim do SRAG com foco na influenza. [acesso em 06 out 2019]. Disponível em: https://extranet.saude. go.gov.br/public/influenza.html

4. Ribeiro SA, Brasileiro GS, Soleiman LNC, Silva CC, Kavaguti CS. Síndrome respiratória aguda grave causada por influenza A (subtipo H1N1). J. bras. pneumol. 
2010;36(3):386-9.

5. Pandemic (H1N1)-update 75. (2009). [acesso em 06 out 2019]. Disponível em: https://www.who.int/ith/ diseases/si_iAh1n1/en/

6. World Health Organization. Influenza seasonal, 6 November, 2018. [acesso em 06 out 2019]. Disponível em: https://www.who.int/news-room/fact-sheets/detail/ influenza-(seasonal)

7. Mondini G, Braga PE, Lopes MH, Sartori AMC, Miyaji KT, Infante $V$ et al. Prospective cohort studies to evaluate the safety and immunogenicity of the 2013,2014 , and 2015 seasonal influenza vaccines produced by Instituto Butantan. Rev. Inst. Med. trop. 2018;60:e37.

8. Neto JC. "Pandemia de Influenza no Brasil: epidemiologia, tratamento e prevenção da Influenza A (H1N1)". Tese de Doutorado em Ciências na área de Epidemiologia em Saúde Pública. Rio de Janeiro: Instituto de Comunicação e Informação Científica e Tecnológica; 2012.

9. Secretaria de Vigilância em Saúde - Ministério da Saúde. Informe Epidemiológico, Influenza: Monitoramento até a Semana Epidemiológica 52 de 2017. [acesso em 06 out 2019]. Disponível em: https://portalarquivos2. saude.gov.br/images/pdf/2018/janeiro/17/InformeEpidemiol--gico-Influenza-2017-SE-52.pdf

10. Guedes ALS, Santos DLS, Júnior ELF, Sousa GT, Vieira NP, Oliveira IP. Análise epidemiológica da situação da influenza pandêmica (h1n1) 2009/2010 no Estado de Goiás. Rev Fac Montes Belos. 2011;4(2)

11. Atlas do Desenvolvimento Humano no Brasil. [acesso em 06 out 2019]. Disponível em: http://www.atlasbrasil. org.br/2013/pt/o_atlas/idhm/

12. Rodríguez-Morales Alfonso J. Asociación entre el desarrolloy la epidemiología de la influenza $\mathrm{A} H 1 \mathrm{~N} 1$ en países de América Latina. Rev. perú. med. exp. salud publica. 2010;27(3):486-7.

13. Montalvão EA. Avaliação de atributos do Sistema de Vigilância Sentinela da Síndrome Gripal no Município do Rio de Janeiro, Brasil, 2013-2014. Dissertação de Mestrado. Rio de Janeiro: Escola Nacional de Saúde Pública Sérgio Arouca, na Fundação Oswaldo Cruz; 2017.

14. Victor JF, Gomes GD, Sarmento LR, Soares AMG, Mota $F R N$, Leite $B M B$, et al. Fatores associados à vacinação contra Influenza A (H1N1) em idosos. Rev. esc. enferm. USP. 2014;48(1):57-64.

15. Oliveira AP. Cobertura da vacina anti-influenza em idosos de municípios do estado do Rio Grande do Sul: 2007-2012. Dissertação de Mestrado. Pelotas: Universidade Federal de Pelotas; 2014.
16. Pereira BFB, Martins MAS, Barbosa TLA, Silva CSO, Gomes LMX. Motivos que levaram as gestantes a não se vacinarem contra H1N1. Ciênc. saúde coletiva. 2013;18(6):1745-52. 